\title{
Solidification in syntectic and monotectic systems
}

\author{
C. Hüter, ${ }^{1}$ G. Boussinot, ${ }^{1,2}$ E. A. Brener, ${ }^{2}$ and R. Spatschek ${ }^{1}$ \\ ${ }^{1}$ Computational Materials Design Department, Max-Planck Institut für Eisenforschung, D-40237 Düsseldorf, Germany \\ ${ }^{2}$ Institut für Festkörperforschung, Forschungszentrum Jülich, D-52425 Jülich, Germany
}

(Received 21 June 2012; published 28 August 2012)

\begin{abstract}
We present theoretical studies of syntectic and monotectic solidification scenarios. Steady-state solidification along the liquid-liquid interface in a syntectic system is considered by means of a boundary-integral technique developed previously. We study the case of small asymmetry of the pattern and extract from the results the scaling relations in terms of the undercooling and the asymmetry parameter. We also investigate monotectic solidification using the phase-field method. We present two kinds of two-phase fingers, with the solid phase being either the exterior phase or the interior phase, and the pattern corresponding to the growth along the solid-liquid interface. We finally analyze the asymptotic shape of these new morphologies far behind their tip.
\end{abstract}

DOI: 10.1103/PhysRevE.86.021603

PACS number(s): 68.08.-p, 64.70.dg

\section{INTRODUCTION}

The occurrence of syntectic transitions is rather rare in established large-scale industrial metallurgical processes. Among the materials which exhibit a syntectic point we mention P-Sn [1], which has relevance for lead-free solders [2], or U- $\mathrm{Pb}$ [3]. In syntectic systems the three-phase equilibrium consists of two liquid phases and one solid phase (see Fig. 1), which is similar in this sense to the monotectic equilibrium. However, unlike the monotectic system, the syntectic one may exhibit a symmetric phase diagram which drastically simplifies the solidification problem. In this paper we study small deviations from this symmetric case in order to extract scaling relations.

The physical processes involved below or above the syntectic temperature include diffusion of solutal elements, convection, and gravity effects. However, we are interested here in the solidification along the liquid-liquid interface in a general picture of three-phase equilibrium in binary alloys (eutectic, peritectic, eutectoid, etc.), and we restrict our study to solutal diffusion, which is assumed to take place only in liquid phases.

We study the solidification problem in the syntectic system, which is initiated by the syntectic reaction at the liquidliquid interface, the subsequent transformation occurring as growth of the solid zone along the liquid-liquid interface [4]. Method of choice is the boundary-integral technique, using the framework developed in Ref. [5] for the solidification of monotectics. This boundary-integral formulation is designed for the modeling of one liquid-liquid and two solid-liquid interfaces. The solidification takes place along the liquid-liquid boundary, and the solid phase appears as a finger-like shape; see Fig. 2.

In the syntectic system, the liquid-liquid mixture is the metastable state below the syntectic temperature for the whole range of concentrations of the syntectic plateau (see Fig. 1). This is different from the monotectic phase diagram, where below the monotectic temperature three possible metastable states exist. In Ref. [5] we studied only the solidification along the liquid-liquid interface. In this work, to complete the study of the phase transition in the monotectic system, we present patterns by the phase-field method which are obtained for the two remaining possible metastable states, i.e., a single liquid phase and a solid-liquid equilibrium.

\section{PHASE DIAGRAM, GEOMETRY, AND BOUNDARY-INTEGRAL FORMULATION}

\section{A. Phase diagram}

At the syntectic temperature the three-phase equilibrium consists of two liquids $L_{1}$ and $L_{2}$ and one solid phase $S$ with concentrations $c_{1}, c_{2}$, and $c_{S}$, respectively. The concentration of the solid phase lies in the interval between the concentration of the two liquids such that $c_{1}<c_{S}<c_{2}$. Above the syntectic temperature, the thermodynamic equilibrium consists of a $\left(L_{1}+L_{2}\right)$ mixture. The liquid-liquid two-phase region spans the whole range of concentrations between $c_{1}$ and $c_{2}$. Below the syntectic temperature the $\left(L_{1}+L_{2}\right)$ mixture is metastable with concentration $c_{12}$ in $L_{1}$ and $c_{21}$ in $L_{2}$ (see Fig. 1). The phase transition is then controlled by the fluxes in $L_{1}$ (whose concentration is $c_{1 S}$ in equilibrium in with $S$ ) and in $L_{2}$ (whose concentration is $c_{2 S}$ in equilibrium in with $S$ ). The concentrations in the solid $S$ are then $c_{S 1}$ in equilibrium with $L_{1}$ and $c_{S 2}$ in equilibrium with $L_{2}$. One defines two control parameters which are the driving forces for classical dendritic solidification $\Delta_{1}=\left(c_{12}-c_{1 S}\right) /\left(c_{S}-c_{1}\right)$ and $\Delta_{2}=$ $\left(c_{2 S}-c_{21}\right) /\left(c_{2}-c_{S}\right)$.

Below the syntectic temperature, three thermodynamic equilibrium domains exist, depending on the concentration of the alloy. If the concentration of the alloy is smaller than $c_{S 1}$, then the $\left(L_{1}+S\right)$ mixture corresponds to the thermodynamic equilibrium. If the concentration of the alloy is larger than $c_{S 2}$, then the thermodynamic equilibrium is the $\left(S+L_{2}\right)$ mixture. If the concentration of the alloy lies in the interval between $c_{S 1}$ and $c_{S 2}$, the thermodynamic equilibrium consists of a single solid phase $S$.

\section{B. Geometry}

A finger-like solid phase $S$ is growing along the $L_{1} / L_{2}$ metastable interface. We consider a two-dimensional pattern with the triple junction as a single point (see Fig. 2). Asymptotically far ahead of the triple junction, the $L_{1} / L_{2}$ interface is aligned with the direction $y$ of the steady-state velocity $v$. The triple junction does not have the same position in the $x$ direction as the asymptotic $L_{1} / L_{2}$ interface, the difference being denoted by $a$. Moreover, at the triple junction, 


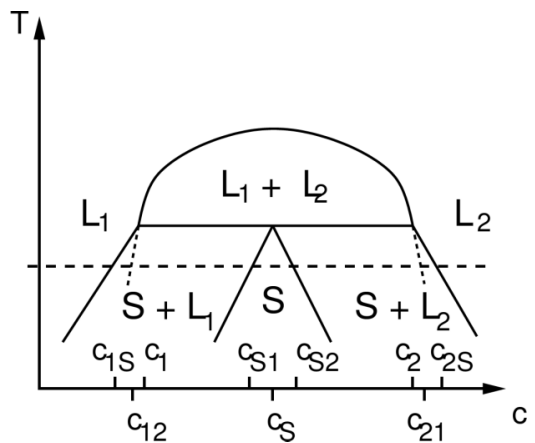

FIG. 1. Phase diagram of the syntectic system.

there is an angle $\delta$ between the $L_{1} / L_{2}$ interface and the growth direction $y$. Far behind the triple junction, the solid-liquid interfaces $S / L_{1}$ and $S / L_{2}$ match separate Ivantsov parabolas [6] of radius $\rho_{1}$ and $\rho_{2}$, respectively. The scaling laws observed in the monotectic alloy [5] and in the eutectic two-phase finger [7] (in a certain limit) suggest that the Ivantsov radius is significantly smaller than the length scale governing the curvatures at the triple junction. The main cancellations of the different terms in the boundary-integral equations operate on a smaller scale than the one needed to match the Ivantsov parabola.

Since we consider an infinite space and since the metastable state consists of a two-phase mixture, the global concentration of the alloy does not enter into the problem. Our results thus do not presuppose any position on the concentration axis on the syntectic plateau (between $c_{1}$ and $c_{2}$ ). However, different cases exist depending on the equilibrium domain where the operating point, i.e., the temperature and the global concentration of the alloy, lies. The ultimate thermodynamic equilibrium is then reached through solid bulk diffusion on scales much larger than the tip region considered here.

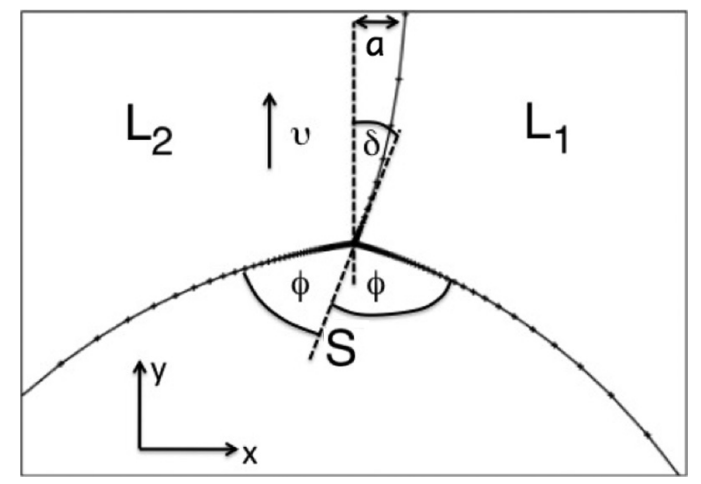

FIG. 2. Close-up of the triple junction region for a typical solution. The solid $S$ grows with velocity $v$ along the metastable liquid-liquid $\left(L_{1} / L_{2}\right)$ interface, which is aligned with $v$ far ahead of the triple junction. In the $x$ direction, the triple junction is shifted by a distance $a$ from the asymptotic position of the liquid-liquid interface. The latter adopts at the triple junction an angle $\delta$ with the velocity direction. The dots indicate the discretization of the interfaces; the vertical asymptotics of the interface $L_{1} / L_{2}$ are satisfied at larger distances to the tip, outside the shown region.

\section{Boundary-integral formulation}

Based on the geometry of the problem and the phase diagram, we obtain the resulting formal presentation of the system. Specifically, we consider steady-state solutions for equal diffusion coefficients in the liquid phases and vanishing diffusion in the growing solid phase. This type of hybrid model, which we first introduced in Ref. [5], thus yields a one-sided model representation [8] of the liquid-solid interfaces and a symmetric model representation of the liquid-liquid interface [9]. The symmetry of the liquid phases also yields equal Gibbs-Thomson corrections to the equilibrium concentrations $c_{12}$ and $c_{21}$. Thus, the solution of the entire problem is reduced to the solution of three coupled integro-differential equations which represent the local equilibria at the different interfaces. The first two of these equations, which are obtained for the solid-liquid interfaces $S / L_{i}(i=1,2)$, read

$$
\frac{1}{2} \frac{c_{i}-c_{S}}{c_{2}-c_{1}}\left\{\Delta_{i}-\frac{d_{0}}{\rho_{1}} \kappa\left[y_{S i}(x)\right]\right\}=I\left[x, y_{S i}(x)\right],
$$

and the third equation, for the $L_{1} / L_{2}$ interface, becomes

$$
-\frac{d_{0}}{\rho_{1}} \kappa\left[x_{12}(y)\right]=I\left[x_{12}(y), y\right] .
$$

The integral $I[x, y]$ which determines the concentration at any point of the space $(x, y)$ reduces to an integration along the three curves $y_{S 1}(x), y_{S 2}(x), x_{12}(y)$, representing the three interfaces $S / L_{1}, S / L_{2}$, and $L_{1} / L_{2}$, respectively. We measure all lengths in units of the Ivantsov radius $\rho_{1}$. The capillary length $d_{0}$ is assumed to be the same on all interfaces without loss of generality, and the curvature is $\kappa[y(x)]=-\left(d^{2} y / d x^{2}\right) /[1+$ $\left.(d y / d x)^{2}\right]^{3 / 2}$. At the triple junction, the liquid-liquid interface obeys $d x_{12} / d y=-\tan (\delta)$, and the self-selected angle $\delta(>0$ in Fig. 2) sets the slopes, precisely $d y_{1 S} / d x=1 / \tan (\phi-\delta)$ and $d y_{2 S} / d x=-1 / \tan (\phi+\delta)$, where $\phi>0$ is the contact angle given by Young's law.

Putting the origin of coordinates at the triple junction, the integral reads

$$
\begin{aligned}
I[x, y]= & \frac{p_{1}}{2 \pi} \frac{c_{2}-c_{S}}{c_{2}-c_{1}} \int_{-\infty}^{0} d x^{\prime}\left[2 g\left(x, y ; x^{\prime}, y_{S 2}^{\prime}\right)\right. \\
& \left.-\left\{\Delta_{2}-\frac{d_{0}}{\rho_{1}} \kappa\left[y_{S 2}\left(x^{\prime}\right)\right]\right\} g^{\prime}\left(x, y ; x^{\prime}, y_{S 2}^{\prime}\right)\right] \\
& +\frac{p_{1}}{2 \pi} \frac{c_{1}-c_{S}}{c_{2}-c_{1}} \int_{0}^{\infty} d x^{\prime}\left[2 g\left(x, y ; x^{\prime}, y_{S 1}^{\prime}\right)\right. \\
& \left.-\left\{\Delta_{1}-\frac{d_{0}}{\rho_{1}} \kappa\left[y_{S 1}\left(x^{\prime}\right)\right]\right\} g^{\prime}\left(x, y ; x^{\prime}, y_{S 1}^{\prime}\right)\right] \\
& +\frac{p_{1}}{\pi} \int_{0}^{\infty} d y^{\prime} g\left(x, y ; x_{12}^{\prime}, y^{\prime}\right) \frac{d x_{12}^{\prime}}{d y^{\prime}}
\end{aligned}
$$

The first two terms come from the integration along the solidliquid interfaces, and we define the Green's function

$$
g\left(x, y ; x^{\prime}, y^{\prime}\right)=\exp \left[-p_{1}\left(y-y^{\prime}\right)\right] K_{0}\left(p_{1} \eta\right)
$$

and its derivative

$$
\begin{aligned}
g^{\prime}\left(x, y ; x^{\prime}, y^{\prime}\right)= & \exp \left[-p_{1}\left(y-y^{\prime}\right)\right] \\
& \times\left[K_{0}\left(p_{1} \eta\right)+f\left(x, y ; x^{\prime}, y^{\prime}\right) K_{1}\left(p_{1} \eta\right)\right],
\end{aligned}
$$


where $p_{1}=\rho_{1} v / 2 D$ is the Péclet number linked to $\rho_{1} . K_{0}\left(K_{1}\right)$ is the modified Bessel function of zeroth (first) order, $\eta=\sqrt{\left(x-x^{\prime}\right)^{2}+\left(y-y^{\prime}\right)^{2}}$, and $f\left(x, y ; x^{\prime}, y^{\prime}\right)=$ $\left[\left(x-x^{\prime}\right) d y^{\prime} / d x^{\prime}-\left(y-y^{\prime}\right)\right] / \eta$.

The driving force $\Delta_{i}$ is related to the Péclet number $p_{i}=v \rho_{i} / 2 D$ by the Ivantsov relation [6],

$$
\begin{aligned}
\Delta_{i} & =\sqrt{\pi p_{i}} \exp \left(p_{i}\right) \operatorname{erfc}\left(\sqrt{p_{i}}\right) \\
& \sim \sqrt{\pi p_{i}}, \quad \Delta_{i} \ll 1 .
\end{aligned}
$$

In order to study the influence of the asymmetry and the undercooling, we rewrite the two solidification driving forces as

$$
\begin{aligned}
& \Delta_{1}=(1-\epsilon) \Delta, \\
& \Delta_{2}=(1+\epsilon) \Delta,
\end{aligned}
$$

where we introduced the asymmetry parameter $\epsilon$, and the undercooling $\Delta$ is proportional to the deviation from the syntectic equilibrium temperature. One should note that the case $\epsilon=0$ corresponds to a fully symmetric pattern where the liquid-liquid interface is the axis of symmetry and the last term on the right-hand side of Eq. (3) vanishes. In opposition to the monotectic system, for which the topology of the phase diagram necessarily implies a strong asymmetry of the pattern (cf. Ref. [5]), we will focus in this work on small deviations from the symmetric pattern, i.e., $\epsilon \ll 1$.

\section{RESULTS: SYNTECTIC SYSTEM}

The solution of the set of equations defined by Eqs. (1) and (2) includes the shapes of the interfaces as well as the values of $d_{0} / \rho_{1}, a / \rho_{1}$, and the angle $\delta$. The transition velocity then is obtained via the Ivantsov relation [Eq. (4)], which implictly gives the Péclet number $p_{i}=v \rho_{i} / 2 D$ for given undercooling. We choose the opening angle $\phi=\pi / 3$ and the miscibility gap ratios $\left(c_{2}-c_{S}\right) /\left(c_{2}-c_{1}\right)=1 / 2,\left(c_{1}-c_{S}\right) /\left(c_{2}-c_{1}\right)=$ $-1 / 2$, which is appropriate for an intuitive definition of the asymmetry parameter. Specifically, as mentioned above, the growth direction becomes the axis of symmetry of the pattern as $\epsilon \rightarrow 0$. A first impression of the results is obtained by the close-up of an exemplary shape in Fig. 2. At the triple junction, the length scale which describes the pattern is the inverse of the curvature close to the triple junction, which we define as $\lambda_{i}=\left(\left.\kappa\left[y_{S i}(x)\right]\right|_{x \rightarrow 0}\right)^{-1}$. In Fig. 3(a) we present the variation of $\lambda_{i} \Delta / d_{0}$ with respect to $\epsilon$, for $\Delta=0.15$ and $\Delta=0.1$. Due to small numerical inaccuracies in the discretization of the integrals (we used about 150 grid points for the interface discretization, which are distributed nonuniformly as shown in Fig. 2), the values of $\lambda_{1} \Delta / d_{0}$ and $\lambda_{2} \Delta / d_{0}$ do not exactly coincide in the limit $\epsilon \rightarrow 0$; In calculations valid only at $\epsilon=0$, this discrepancy is reduced, and the common value $\lambda \Delta / d_{0}$ is approximately the average of $\lambda_{i} \Delta / d_{0}$ at $\epsilon=0$. One clearly sees that $\lambda_{1} \Delta / d_{0}$ and $\lambda_{2} \Delta / d_{0}$ have a linear variation with opposite slopes of about \pm 3.5 . In Fig. 3(b) we plot $\lambda \Delta / d_{0}$ versus $\Delta$ for $\epsilon=0$, with $\lambda=\lambda_{i}(\epsilon=0)$. We clearly see that in the limit $\Delta \rightarrow 0, \lambda \Delta / d_{0}$ is constant. The information provided by Figs. 3(a) and 3(b) suggests that the inverse of the curvatures of the solid-liquid interfaces at the triple junction $\lambda_{i}(\Delta, \epsilon)$ obeys a scaling law given by $\lambda_{i} / \lambda-1 \sim \epsilon$ with $\lambda / d_{0} \sim 1 / \Delta$ in the regimes $\Delta \ll 1, \epsilon \ll 1$.
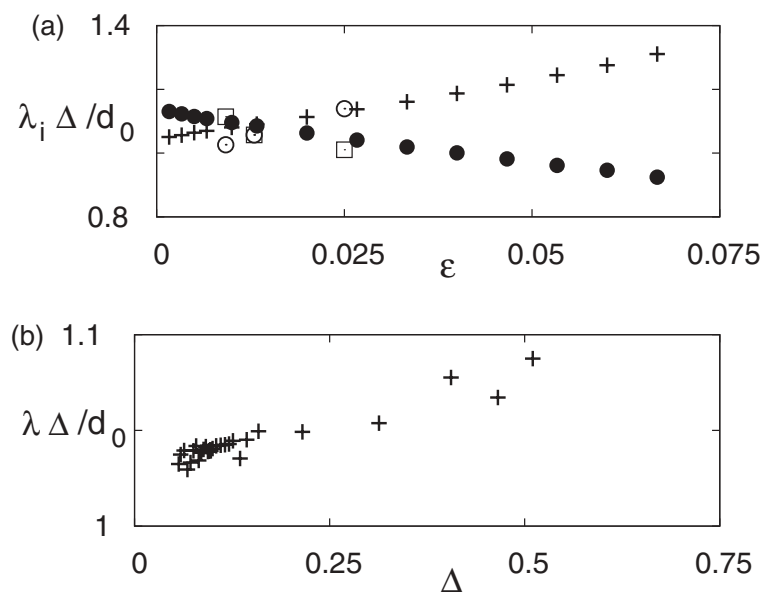

FIG. 3. (a) Linear dependence of $\lambda_{i} \Delta / d_{0}$ with respect to the asymmetry parameter $\epsilon$. For $\Delta=0.15$ crosses indicate $i=1$, filled circles $i=2$, for $\Delta=0.1$, open circles $i=1$, and boxes $i=2$. The numerical inaccuracy explained above briefly is responsible for the slight difference between $\lambda_{1} \Delta / d_{0}$ and $\lambda_{2} \Delta / d_{0}$ when $\epsilon \rightarrow 0$. (b) Plot of $\lambda \Delta / d_{0}$ versus driving force $\Delta$ for $\epsilon=0$, where $\lambda$ is the common value of $\lambda_{1}$ and $\lambda_{2}$.

Concerning the rotation angle $\delta$, we present in Fig. 4 its variation with respect to $\epsilon$ for $\Delta=0.15$ and $\Delta=0.10$. We clearly see that $\delta$ increases linearly with $\epsilon$, at the same time being independent of $\Delta$.

Concerning the asymptotic lateral shift of the liquid-liquid interface $a$ as $y \rightarrow \infty$, we present the quantity $a \Delta / d_{0}$ versus $\epsilon$ in Fig. 5 for $\Delta=0.15$ and $\Delta=0.10$. The figure clearly suggests that the scaling of $a$ is $a / d_{0} \sim \epsilon / \Delta$ in the regime $\Delta \ll 1, \epsilon \ll 1$.

The remaining unknown is the velocity $v$, which is related to $\rho_{i}$ by the Ivantsov relation [Eq. (4)]. In Fig. 6 where $v d_{0} / 2 D$ is given versus $\epsilon$ for $\Delta=0.15$, we see that the velocity is independent of $\epsilon$ in the range of investigated values of $\epsilon$. One should note that the absence of a linear dependence of the velocity on $\epsilon$ can be understood by symmetry; i.e., changing the sign of $\epsilon$ should not change the value of the velocity. Therefore, the first variation of $v$ is of order $\epsilon^{2}$, which is weak in the range of $\epsilon$ presented in Fig. 6 .

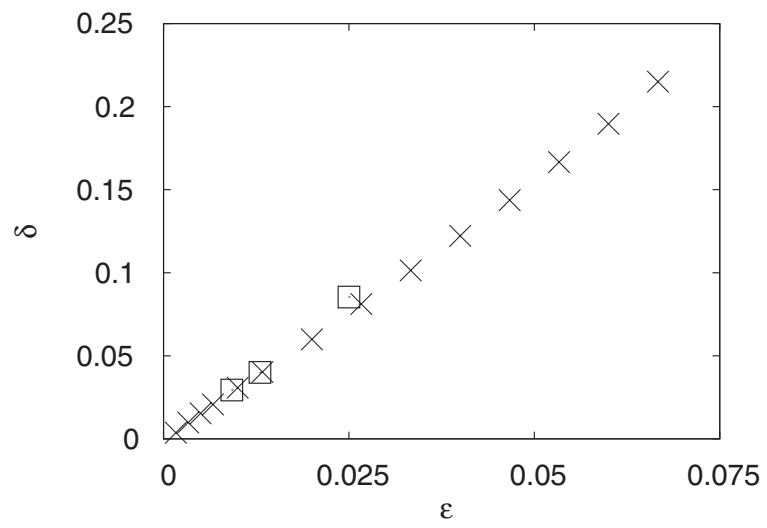

FIG. 4. The rotation angle of the triple junction $\delta$. The crosses correspond to $\Delta=0.15$, the boxes to $\Delta=0.1$. 


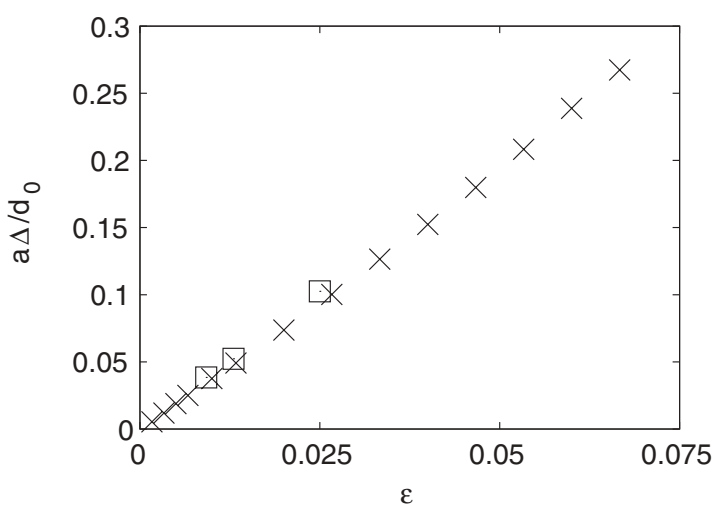

FIG. 5. The ratio $a \Delta / d_{0}$ as function of the asymmetry parameter $\epsilon$. The crosses correspond to $\Delta=0.15$ and the boxes to $\Delta=0.1$.

The dependence of $v$ on $\Delta$ is illustrated in Fig. 7 through the plot of $v \lambda^{2} / 2 d_{0} D$ versus $\Delta$ for $\epsilon=0$. We recall that $\lambda$ is the common value of $\lambda_{1}$ and $\lambda_{2}$ for $\epsilon=0$. We see that $v \lambda^{2} / 2 d_{0} D$ is independent of $\Delta$ for $\Delta \ll 1$. The invariance of this quantity reminds one of the case of classical dendritic [8] or eutectic growth $[7,10]$. Consequently, we obtain the velocity scaling $v d_{0} / 2 D \sim \Delta^{2}$ in the regime $\Delta \ll 1, \epsilon \ll 1$. Since $v d_{0} / 2 D=p_{1} d_{0} / \rho_{1} \sim \Delta_{1}^{2} d_{0} / \rho_{1}=(1-\epsilon)^{2} \Delta^{2} d_{0} / \rho_{1}$ in the regime $\Delta \ll 1$, we have $v d_{0} / 2 D \sim(1-2 \epsilon) \Delta^{2} d_{0} / \rho_{1}$ and therefore $d_{0} / \rho_{1} \sim 1+2 \epsilon$ in the regime $\Delta \ll 1, \epsilon \ll 1$. By the same arguments, we have $d_{0} / \rho_{2} \sim 1-2 \epsilon$. This means that $\rho_{i} / \lambda \rightarrow 0$ for $\Delta \rightarrow 0$, demonstrating the existence of intermediate asymptotics as previously mentioned in Ref. [5] and in Sec. II B. However, the intermediate asymptotics are not the focus of this article.

We summarize the obtained results for the syntectic solidification by the scaling laws for the regime $\Delta \ll 1, \epsilon \ll 1$ :

$$
\frac{\lambda_{i}}{\lambda}-1 \sim \epsilon ; \quad \frac{\lambda}{d_{0}} \sim \frac{1}{\Delta} ; \quad \frac{a}{d_{0}} \sim \frac{\epsilon}{\Delta} ; \quad \delta \sim \epsilon ; \quad \frac{v d_{0}}{D} \sim \Delta^{2} .
$$

\section{RESULTS: MONOTECTIC SYSTEM}

We present now in this part a phase-field study of the isothermal solidification in the monotectic system that complements our previous study [5]. We adapted a phase-field

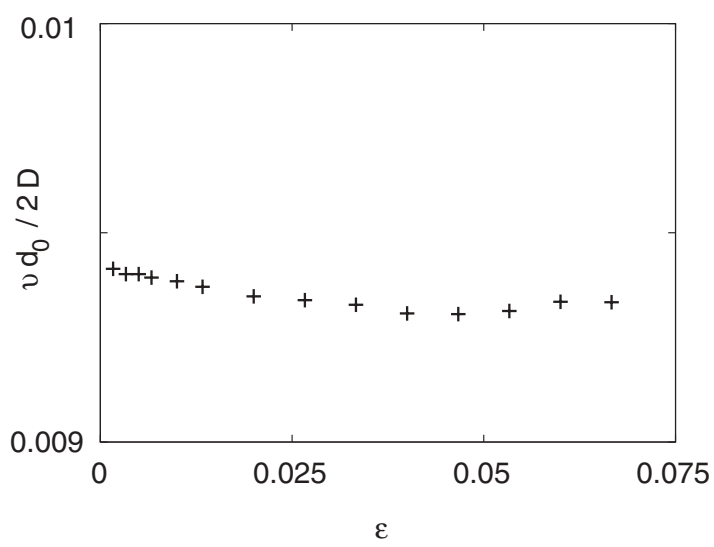

FIG. 6. The dimensionless velocity $v d_{0} / 2 D$ versus $\epsilon$ for $\Delta=0.15$.

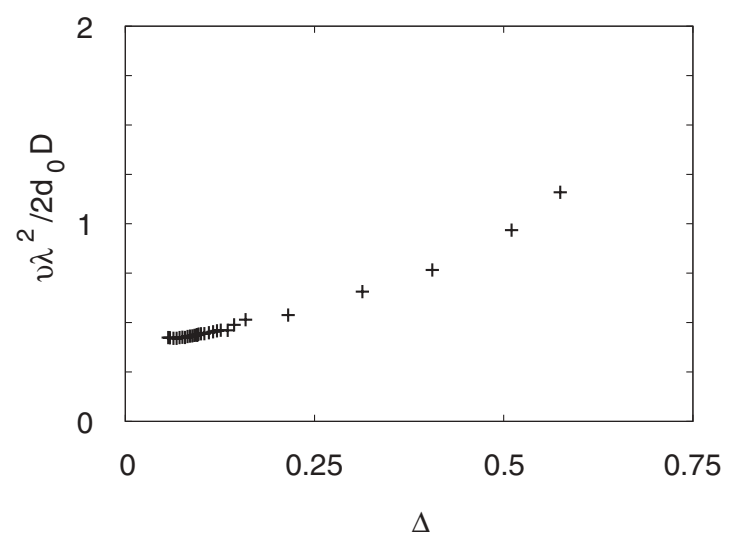

FIG. 7. The invariant $v \lambda^{2} / 2 d_{0} D$ of the syntectic solidification versus $\Delta$ for $\epsilon=0$.

model initially developed for the solidification of eutectic or peritectic alloys [11] in order to account for diffusion in the two liquid phases. In Fig. 8 the monotectic phase diagram is shown. As mentioned in the introduction, the liquid-liquid equilibrium does not span the whole monotectic plateau in the monotectic system. One has three possible metastable states below the monotectic temperature (which are stable above the monotectic temperature) depending on the global concentration of the alloy: a $\left(L_{1}+L_{2}\right)$ liquid-liquid mixture, a single $L_{1}$ liquid phase, and a $\left(S+L_{1}\right)$ solid-liquid mixture. We present in the following phase-field calculations of the phase transitions occurring for a single metastable $L_{1}$ phase and a metastable $\left(S+L_{1}\right)$ mixture.

\section{A. Metastable liquid phase $L_{1}$}

When the global concentration of the alloy is in the neighborhood of the monotectic point, the metastable state consists of a single $L_{1}$ phase. In this region of the phase diagram, the growth of the solid $S$ and the liquid $L_{2}$ may proceed through a two-phase finger growth. The latter has been described recently [7] in the eutectic system. The pattern then consists of one growing solid phase exhibiting Ivantsov asymptotics (exterior phase) surrounding the other solid phase (interior phase). Since diffusion is neglected in the solid phases, the interior phase is a straight lamella parallel

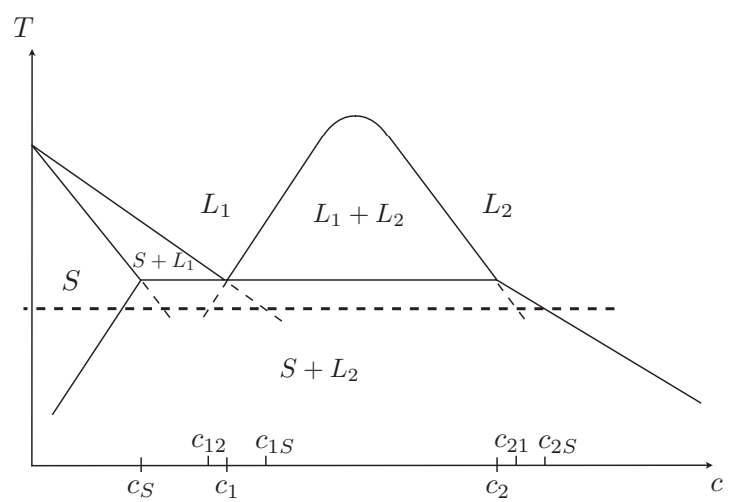

FIG. 8. Monotectic phase diagram. The metastable continuation of the boundaries of the $\left(L_{1}+L_{2}\right)$, and the $\left(S+L_{1}\right)$ equilibrium domains are shown as dashed lines. 
to the direction of growth. In the monotectic system, the two-phase finger can be of two kinds. The exterior phase can be the solid phase $S$ or the liquid phase $L_{2}$. In the present section, we present phase-field simulations of the two patterns.

The fluxes in the neighborhood of the triple junction are described by two dimensionless parameters $\Delta_{1}=\left(c_{1 S}-c_{12}\right)$ / $\left(c_{1}-c_{S}\right)$ and $\Delta_{2}=\left(c_{2 S}-c_{21}\right) /\left(c_{2}-c_{S}\right)$ (see Fig. 8). Depending on which phase is the exterior phase, the global concentration of the alloy $c_{\infty}$ enters into the expression for a dimensionless solidification driving force, written in the standard form for the classical dendrite as $\Delta_{\infty}^{S}=\left(c_{1 S}-c_{\infty}\right) /\left(c_{1}-c_{S}\right)$ when the exterior phase is $S$ and $\Delta_{\infty}^{L}=\left(c_{12}-c_{\infty}\right) /\left(c_{1}-c_{2}\right)$ when the exterior phase is $L_{2}$.

\section{Two-phase finger with a solid exterior phase}

In this case, the pattern is close to the one obtained in eutectic systems, and the exterior solid phase exhibits Ivantsov asymptotics. In Fig. 9(a) we present the corresponding pattern, where the growth direction is indicated by the arrow. The ratio of miscibility gaps at equilibrium is $\left(c_{1}-c_{S}\right) /\left(c_{2}-c_{1}\right)=$ 0.86 . The solidification driving forces are $\Delta_{1}=0.252$ and $\Delta_{2}=0.0540$ (see Fig. 8). The concentration $c_{\infty}$ of the liquid $L_{1}$ far ahead of the tip of the pattern is such that $\Delta_{\infty}^{S}=0.225$ and $\Delta_{\infty}^{L} \simeq \Delta_{\infty}^{S} / 10$. The selected pattern with the solid phase as the exterior phase thus corresponds to the largest driving force $\Delta_{\infty}^{S}$. Here no flux exists in the liquid $L_{2}$ far behind the tip, and the $S / L_{2}$ interface is parallel to the growth direction. The lateral position (perpendicular to the growth direction) of the $S / L_{2}$ interface with respect to the axis of symmetry of the pattern is $a=18.2 d_{0}$, and the dimensionless velocity is $v d_{0} / 2 D=4.125 \times 10^{-3}$. Therefore the quantity $a^{2} v / 2 D \simeq 1.37$ is of order unity.

In Fig. 9(b) we present a phase-field simulation of an oscillating two-phase finger. Here we have $\left(c_{1}-c_{S}\right) /\left(c_{2}-\right.$ $\left.c_{1}\right)=0.46$, the driving forces are $\Delta_{1}=0.088, \Delta_{2}=0.1 \Delta_{1}$, and $\Delta_{\infty}^{S}=0.065\left(\Delta_{\infty}^{L} \simeq \Delta_{\infty}^{S} / 6\right)$. The total width $2 a$ of the $L_{2}$ phase perpendicular to the growth direction oscillates between $60 d_{0}$ and $105 d_{0}$, and the dimensionless velocity averaged over one period is $v d_{0} / 2 D=2.18 \times 10^{-4}$. Thus the quantity $a^{2} v / 2 D$ oscillates between 0.2 and 0.66 and is of order unity again. This oscillating pattern reminds one of the oscillatory

(a)

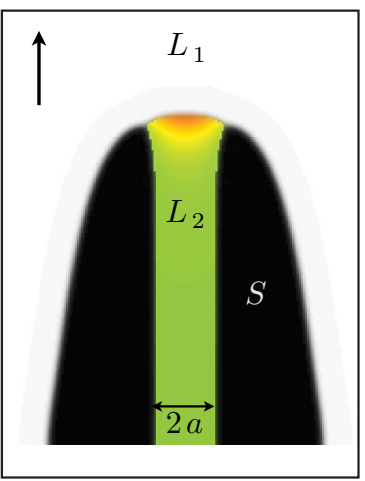

(b)

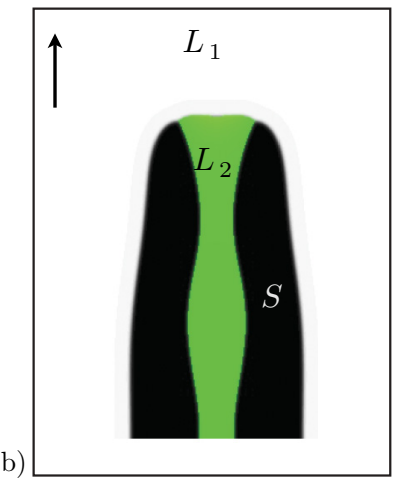

FIG. 9. (Color online) Two-phase finger with the solid (black) surrounding the liquid $L_{2}$ (green/gray) in (a) steady state and (b) an oscillatory regime. The arrow indicates the growth direction. regime in lamellar growth in eutectics (we actually obtained the same kind of oscillatory regime previously during the growth of eutectic two-phase fingers in the framework of phase-field simulations) $[12,13]$. However, one should not exclude the possibility of an extremely slow decay of the oscillations towards a steady state. We observed at least ten periods without damping of the oscillations of the velocity. One should also not exclude an artificial stabilization of the oscillatory regime when the interface width of the phase fields [11] (here about $0.2 a$ ) is not well separated from the length scales of the pattern.

\section{Two-phase finger with liquid exterior phase}

In this second case, the liquid $L_{2}$ surrounds the solid phase $S$, and far behind the tip we have a $L_{2}$-film-migration process (LFM) [14,15], i.e., the combined motion of two parabolic fronts enclosing the $L_{2}$ phase. In Fig. 10 we present this pattern. It was obtained when $\left(c_{1}-c_{S}\right) /\left(c_{2}-c_{1}\right)=2, \Delta_{1}=0.105$, $\Delta_{2}=0.0467$, and for $c_{\infty}=c_{1}$. Then we have $\Delta_{\infty}^{L}=0.167$ and $\Delta_{\infty}^{S} \simeq \Delta_{\infty}^{L} / 8$. Therefore, the obtained pattern with the liquid phase as exterior phase is the one that corresponds to the largest driving force $\Delta_{\infty}^{L}$. The dimensionless velocity is $v d_{0} / 2 D=1.75 \times 10^{-3}$, and the lateral position of the triple junction with respect to the axis of symmetry of the finger is $a=16.5 d_{0}$. Therefore the quantity $a^{2} v / 2 D=0.48$ is here also of order unity.

Let us make a comment on the LFM process that occurs here. In Ref. [14] the LFM has been theoretically studied, and the Péclet numbers $P_{S}=v R_{S} / 2 D$ and $P_{2}=v R_{2} / 2 D$ of the two parabolic fronts $S / L_{2}$ and $L_{2} / L_{1}$, respectively, of radius $R_{S}$ and $R_{2}$, have been determined depending on the control parameters $\Delta_{2}$ and $\Delta_{\infty}^{L}$. In the limit $P_{S}, P_{2} \ll 1$, one has

$$
\begin{aligned}
& P_{S}=\frac{\Delta_{2} / 2}{\lambda-1+\chi+\sqrt{\chi^{2}+2(\lambda-1) \chi}}, \\
& P_{2}=P_{S}\left[\lambda+\chi+\sqrt{\chi^{2}+2(\lambda-1) \chi}\right]^{2}
\end{aligned}
$$

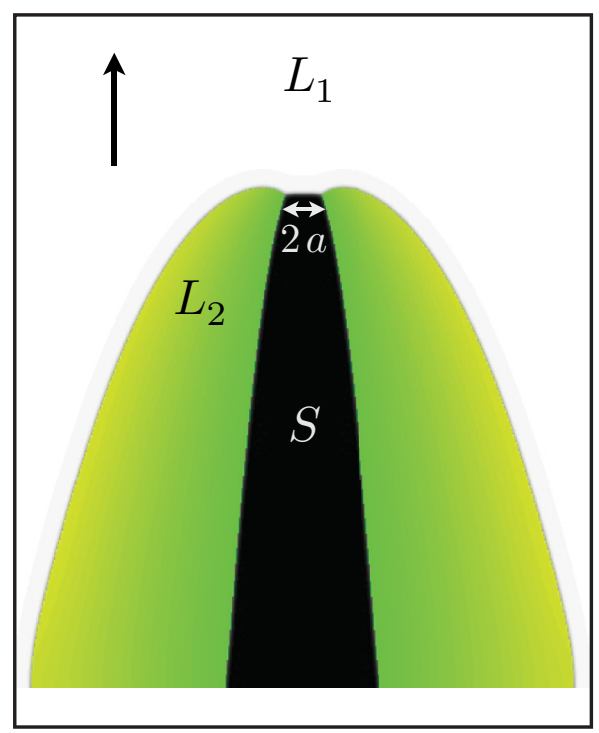

FIG. 10. (Color online) Two-phase finger growth with the liquid $L_{2}$ (green/gray) surrounding the solid $S$ (black). Far behind the tip, one has a $L_{2}$-film migration process. 


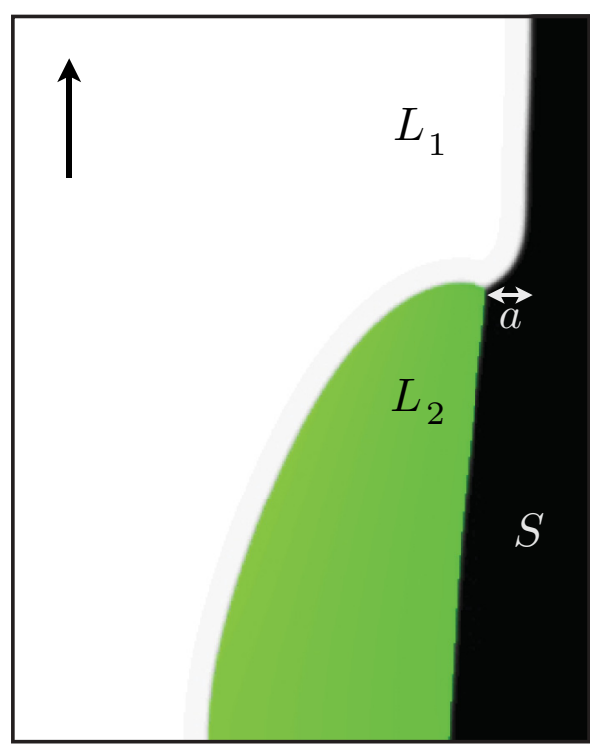

FIG. 11. (Color online) Growth along the $S / L_{1}$ interface. Far behind the tip, one has a $L_{2}$-film migration process.

where $\lambda=\left(c_{2}-c_{S}\right) /\left(c_{2}-c_{1}\right)>1$ and $\chi=\left(\Delta_{\infty}^{L}\right)^{2} /\left(\pi \Delta_{2}\right)$. Here $\lambda=3$ and $\chi=0.190$. Therefore the ratio $P_{2} / P_{S} \simeq 10$ is large, and a quantitative comparison with the theory is difficult. More precisely, the size of the simulated portion of the pattern does not allow for an accurate determination of the curvature of the $S / L_{2}$ front.

It is interesting to note that $\chi$, which tunes the ratio $P_{2} / P_{S}$, has the same form as the quantity that determines the ratio between the width of the interior lamella and the Ivantsov radius for the growth of the two-phase finger in the eutectic system [7]. In the case $\chi=0$, i.e., $\Delta_{\infty}^{L}=0$, one has $P_{S}=\Delta_{2} /(2 \lambda-2)$ and $P_{2} / P_{S}=\lambda^{2}$. This result has been presented for the LFM process above the peritectic temperature in Ref. [16]. In the other limit $\chi \rightarrow \infty, P_{2} / P_{S} \rightarrow 4 \chi^{2}$, and one recovers the Ivantsov relation for the $L_{1} / L_{2}$ interface; i.e., $P_{2} \simeq\left(\Delta_{\infty}^{L}\right)^{2} / \pi$ becomes independent of $\Delta_{2}$.

\section{B. Metastable solid-liquid mixture $\left(S+L_{1}\right)$}

When a $\left(S+L_{1}\right)$ mixture is brought into a metastable state below the monotectic temperature, the transformation consists of the growth of the solid $S$ and the liquid $L_{2}$ along the metastable $S / L_{1}$ interface. In Fig. 11 we present the corresponding pattern. The $S / L_{1}$ interface is aligned with the direction of growth far ahead of the triple junction and adopts an angle in its neighborhood. Far behind the triple junction, one has again a $L_{2}$-film migration process.
This pattern is close to the one described in Ref. [17] for the melting of a peritectic alloy along the solid-solid interface. However, here a lateral shift $a$ (perpendicular to the growth direction) of the triple junction with respect to the asymptotic position of the metastable interface exists contrary to the case where the metastable interface separates two solid phases. The miscibility gaps are such that $\left(c_{1}-c_{S}\right) /\left(c_{2}-c_{1}\right)=2$, and the driving forces are $\Delta_{1}=0.0524$ and $\Delta_{2}=0.0233$. The concentration in $L_{1}$ far ahead of the triple junction is $c_{\infty}=c_{1 S}$, and thus $\Delta_{\infty}^{L}=\Delta_{1}\left(c_{1}-c_{S}\right) /\left(c_{2}-c_{1}\right)=0.105$ and $\Delta_{\infty}^{S} \simeq 0$. Hence we have $\chi=0.150$ for the description of the LFM process. Again $\lambda=3$, and the size of the simulated portion of the pattern does not allow one to calculate accurately the ratio $P_{2} / P_{S}$.

\section{SUMMARY}

In this paper we have presented a study of the solidification in the syntectic system applying a boundary integral technique developed in our previous work [5] and a study of different solidification scenarios in monotectic systems using the phasefield method.

In the syntectic system we have focused on small deviations from the symmetric pattern, corresponding to small values $\epsilon \ll 1$ of an asymmetry parameter $\epsilon$. We have extracted from our boundary integral calculations the scaling relations of the relevant length scales, the rotation angle of the triple junction $\delta$, and the growth velocity, which are summarized in Eq. (7).

For the monotectic system we have presented phase-field simulations of scenarios of solidification complementary to the solidification along the liquid-liquid interface presented in Ref. [5]. First, we have studied the two-phase fingers growing at the expense of a single metastable liquid phase. We have obtained a two-phase finger with the solid phase as the exterior phase which is close to the eutectic finger presented in Ref. [7], and in addition we have shown the possibility of oscillatory regimes of this mode of growth. The existence of these nonsteady state patterns is the reason why we have employed phase field instead of boundary-integral methods for the solution of this problem. We have obtained also a two-phase finger where the solid phase is the interior phase, and the asymptotics far behind the tip of the pattern correspond to a liquid-film-migration process. Second, we have studied the solidification along the solid-liquid interface, which exhibits the same liquid-film-migration asymptotic behavior.

\section{ACKNOWLEDGMENT}

We are grateful to M. Plapp for providing his phase-field code.
[1] H. Okamoto, J. Phase Equilib. 14, 263 (1993).

[2] K. Subramanian, Lead-Free Solders (John Wiley and Sons, New York, 2012).

[3] R. J. Teitel, J. Metals Trans. AIME 4, 397 (1952).

[4] S. P. Gupta, Phase Equilibria in Materials (Allied Publishers Pvt. Ltd., New Delhi, 2003).
[5] C. Hüter, G. Boussinot, E. A. Brener, and D. E. Temkin, Phys. Rev. E 83, 050601(R) (2011).

[6] G. P. Ivantsov, Dokl. Akad. Nauk USSR 58, 567 (1947).

[7] G. Boussinot, C. Hüter, and E. A. Brener, Phys. Rev. E 83, 020601(R) (2011). 
[8] Y. Saito, G. Goldbeck-Wood, and H. Müller-Krumbhaar, Phys. Rev. Lett. 58, 1541 (1987).

[9] J. S. Langer and L. A. Turski, Acta Metal. 25, 1113 (1977).

[10] K. Kassner and C. Misbah, Phys. Rev. Lett. 66, 445 (1991).

[11] R. Folch and M. Plapp, Phys. Rev. E 72, 011602 (2005).

[12] A. Karma and A. Sarkissian, Metal. Trans. A 27, 635 (1996).
[13] M. Ginibre, S. Akamatsu, and G. Faivre, Phys. Rev. E 56, 780 (1997).

[14] D. E. Temkin, Acta Mat. 53, 2733 (2005).

[15] E. A. Brener and D. E. Temkin, Phys. Rev. Lett. 94, 184501 (2005).

[16] G. Boussinot, E. A. Brener, and D. E. Temkin, Acta Mat. 58, 1750 (2010).

[17] E. A. Brener and D. E. Temkin, Acta Mat. 55, 2785 (2007). 